\title{
An arc partition of the Hughes plane using a field-theoretic model
}

go back

full screen

close

quit

\author{
Ronald D. Baker Kenneth L. Wantz
}

\begin{abstract}
Nearfield models for PG $\left(2, q^{2}\right)$ and the Hughes plane of order $q^{2}$, based on the well-known field-theoretic model of $\mathrm{PG}\left(2, q^{2}\right)$, are described. By way of the correspondences between these models, certain unitals, ovals, and a Baer subplane are easily described in the Hughes plane. Moreover, a partition of the Hughes plane into maximal $\left(q^{2}-q+1\right)$-arcs is presented.
\end{abstract}

Keywords: nearfield, arc partition, Hughes plane

MSC 2000: 51E20, 51E21

\section{Introduction}

When modelling projective planes using coordinates, one can easily get confused by the well-known results relating the algebraic properties of the planar ternary rings (PTR's) with the types of planes, as there are other ways that algebraic systems can be used to model projective planes. In particular, the Hughes plane arises from a nearfield, but is not coordinatized by any PTR. In light of this, one should not be surprised that the Desarguesian plane can also be modelled using the same nearfield as that used to construct the Hughes plane. In this paper we model both planes using a nearfield and exploit these models to develop some configurations in the Hughes plane that exist because of this close connection. Additionally, we hope that this model will provide a straightforward construction of the Hughes plane; one particularly well-suited for use with the built-in finite field tools of computer algebra systems.

Let $F$ be a finite field of odd order $q^{6}$ and let $F_{S}$ and $F_{N}$ denote the sets of squares and nonsquares of $F$, respectively. Further let $K$ denote the subfield of $F$ of order $q^{2}$, with $K_{S}$ and $K_{N}$ similarly defined. Additionally, we define 



\section{Nearfield models}

A nearfield model of PG $\left(2, q^{2}\right)$ will be presented first. Recall that the nearfield $N$ has order $q^{6}$ and is a three-dimensional module over $N_{0}$. The one-dimensional submodules of $N$ over $N_{0}$ are used to represent the points of $\mathrm{PG}\left(2, q^{2}\right)$ in this model. Any nonzero element of $N$ belongs to a unique one-dimensional submodule, so nonzero elements can be used to represent the $q^{4}+q^{2}+1$ points. Moreover, for $u, v \in N^{*}=N \backslash\{0\}, u$ and $v$ represent the same point if and only if $u=c \cdot v$ for some $c \in N_{0}^{*}$. We denote this equivalence relation on $N^{*}$ by $\sim$.

As is typically the case in models involving homogeneous coordinates, a canonical representative of each point is useful. Our preference for representing points is to choose an element of $F_{S}$ as this will allow us to establish an isomorphism between the two models of the Desarguesian plane and to establish a correspondence with the model of the Hughes plane. Specifically, for $\beta$ a primitive element of $F$, the following proposition shows that $\beta^{2 i}$ for $i=0,1, \ldots, q^{4}+q^{2}$ serve as distinct representatives for the points of $\mathrm{PG}\left(2, q^{2}\right)$ in the nearfield model.

Proposition 2.1. Let $\beta$ be a primitive element of $F=\operatorname{GF}\left(q^{6}\right)$ with $i$ and $j$ integers. Then $\beta^{2 i} \sim \beta^{2 j}$ implies $i \equiv j\left(\bmod q^{4}+q^{2}+1\right)$.

Proof. First note that $a \cdot v$ is a square in $F$ if and only if $a \in K_{S}$ and $v \in F_{S}$. Now if $\beta^{2 i} \sim \beta^{2 j}$, then $\beta^{2 i}=a \cdot \beta^{2 j}$ where $a$ is necessarily an element of $K_{S}$. Therefore, $\beta^{2 i}=a \beta^{2 j}$ and $a=\beta^{2(i-j)}$. Furthermore, as $\beta^{q^{4}+q^{2}+1}$ is primitive in $K, a=\beta^{\left(q^{4}+q^{2}+1\right) 2 k}$ for some integer $k$. As 2 is relatively prime to $q^{4}+q^{2}+1$, the result holds.

The lines of $\mathrm{PG}\left(2, q^{2}\right)$ in this model are defined for $v \in F^{*}$ as $\{u \in F$ : $\operatorname{Tr}(u \cdot v)=0\}$ and incidence of a point with a line is given by inclusion. To see that this incidence is well-defined, suppose $\operatorname{Tr}(u \cdot v)=0$ and $c \in N_{0}^{*}$. Then $\operatorname{Tr}((c \cdot u) \cdot v)=\operatorname{Tr}(c \cdot(u \cdot v))$, and if $c \in F_{S}$ this equals $\operatorname{Tr}(c(u \cdot v))=c \operatorname{Tr}(u \cdot v)=0$, and if $c \in F_{N}$ this equals $\operatorname{Tr}\left(c(u \cdot v)^{q^{3}}\right)=c \operatorname{Tr}(u \cdot v)^{q}=0$. Therefore the incidence relation is independent of the point representative, and we see that a line may be viewed as a collection of one-dimensional submodules. Furthermore, the lines are representable as one-dimensional vector subspaces of $F$ over $K$, since for $k \in K^{*}, \operatorname{Tr}(u \cdot(k v))$ equals $\operatorname{Tr}(u(k v))=k \operatorname{Tr}(u v)=k \operatorname{Tr}(u \cdot v)=0$ when $u \in F_{S}$ and equals $\operatorname{Tr}\left(u(k v)^{q^{3}}\right)=k^{q} \operatorname{Tr}\left(u v^{q^{3}}\right)=k^{q} \operatorname{Tr}(u \cdot v)=0$ when $u \in F_{N}$.

To see that this incidence structure is a projective plane and a model of $\mathrm{PG}\left(2, q^{2}\right)$, we note that using the point-representatives from $F_{S}$ as in the above proposition, $\operatorname{Tr}\left(\beta^{2 i} \cdot v\right)$ is simply $\operatorname{Tr}\left(\beta^{2 i} v\right)$. Thus a given line represented by $v$ in 








\section{References}

[1] R. D. Baker, G. L. Ebert, G. Korchmáros, and T. Szönyi, Orthogonally divergent spreads of Hermitian curves. In Finite Geometry and Combinatorics, F. De Clerck et al editor, Cambridge Univ. Press (1993), 17-30.

[2] R. D. Baker and K. L. Wantz, Unitals in the code of the Hughes plane. $J$. Combin. Designs 12(1) (2004), 35-38.

[3] E. Boros and T. Szönyi, On the sharpness of a theorem by B. Segre. Combinatorica 6(3) (1986), 261-268.

[4] J. C. Fisher, J. W. P. Hirschfeld, and J. A. Thas, Complete arcs in planes of square order. Annals of Discrete Math. 30 (1986), 243-250.

[5] D. R. Hughes, A class of non-desarguesian projective planes. Canad. J. Math. 9 (1957), 378-388.

[6] D. R. Hughes and F. C. Piper, Projective planes. Springer-Verlag, New York, 1973.

[7] L. A. Rosati, Disegni unitari nei piani di Hughes. Geom. Ded. 27 (1988), 295-299.

[8] K. L. Wantz, A new class of unitals in the Hughes plane. Geom. Ded. 70(2) (1998), 125-138.

Ronald D. Baker

Department of Mathematics, West Virginia State University, Institute, West Virginia 25112

e-mail: baker@ehmoore.wvstateu.edu

Kenneth L. Wantz

Department of Mathematics, Southern NAZAREne University, Bethany, OKLAhoma 73008

e-mail: kwantz@snu.edu 